\title{
Free Entanglement Measure of Multiparticle Quantum States
}

\author{
Chang-shui $\mathrm{Yu}^{*}$, He-shan Song \\ Department of Physics, Dalian University of Technology, Dalian 116024, China
}

\begin{abstract}
In this paper, based on the classfication of multiparticle states and the original definition of semiseparability, we give out the redefinition of semiseparability and inseparability of multiparticle states. By virtue of the redefinition, entanglement measure of multiparticle states can be converted into bipartite entanglement measure in arbitrary dimension in mathematical method. A simple expression of entanglement measure is given out. As examples, a general three-particle pure state and an N-particle mixed state are considered.
\end{abstract}

Key words: entanglement measure, multiparticle entanglement, free entanglement PACS: 03.67.-a, 03.65.-Ta

\section{Introduction}

Entanglement as a valuable resource has been widely applied to quantum communication and quantum information processing. Quantum teleportation [1], entanglement swapping [2], quantum key distribution [3] and quantum correction and so on make use of quantum entanglement, the profoundly important resource, in essence. Therefore, the quantification of entanglement as a central problem in quantum information theory is a primary goal of this field.

Quantum entanglement has attracted a lot of attention in recent years. A lot of studies of quantum entanglement has been proposed and at the same time, many entanglement measures come up [4-9]. However, only bipartite entanglement with two levels has been perfectly complished [5], and there are a lot of open questions in quantifying entanglement for the bipartite entanglement

* Corresponding author.quaninformation@sina.com 
measure in arbitrary dimensions and the multiparticle entanglement measure. Fortunately, the method for classifying a three-particle state [10] and the one presented recently for quantifying the bipartite entanglement in arbitrary dimension [11] would increase our understanding of multiparticle entanglement.

For some entanglement measure, there must exist a corresponding separability criterion. However, so far there have not been an operational multiparticle full separability criterion, but only a semiseparability definition [12]. Hence it is very difficult to obtain a thorough entanglement measure for multiparticle systems.

In this paper, we study multiparticle free entanglement measure [12] with a new idea(Strictly, free entanglement here denotes the entanglement of states which excludes the fourth class defined in [10], and for convenience, free entanglement is substituted by entanglement for multiparticle systems later). Based on the classfication of multiparticle states, we express semiseparability condition and full inseparability condition of multiparticle systems in a unified way. By virtue of the conditions, we find some mathematical counterpart of entanglement and convert multiparticle entanglement measure into bipartite entanglement in arbitrary dimension in mathematics. Then we give out a simple multipartitle entanglement measure according to the bipartite quantum entanglement measure. Finally, we give an example demonstrating our measure can work effectively in its right for pure states and mixed states respectively.

\section{$2 \quad$ Separability and Inseparability}

We begin with the usual definition of multiparticle entanglement. For $N$ particle pure state $\left|\Psi^{A B C \cdots N}\right\rangle$, if it can be written in the form of direct product of all the subsystems, i.e.

$$
\left|\Psi^{A B C \cdots N}\right\rangle=\left|\psi^{A}\right\rangle \otimes\left|\psi^{B}\right\rangle \otimes \cdots \otimes\left|\psi^{N}\right\rangle
$$

then $N$-particle pure state is separable; If $N$-particle mixed state $\rho^{A B C \cdots N}$ is separable, the state can be written in the following form:

$$
\rho^{A B C \cdots N}=\sum_{i} p_{i}\left|\psi_{i}^{A}\right\rangle\left\langle\psi_{i}^{A}|\otimes \cdots \otimes| \psi_{i}^{N}\right\rangle\left\langle\psi_{i}^{N}\right|
$$

where $\sum_{i} p_{i}=1, p_{i}>0$ and $\psi_{i}^{\alpha}$ with $i=0,1, \cdots$, is any normalized state of the subsystem $\alpha$. Hence, if any multiparticle state cannot be written in the above forms, the state is called an entangled state. However, the definition per se is not operational, we have to turn to an operational one. 
Three-particle states can be classified according to whether they are seperable or not with respect to the different qubits [10]. They can be classified into five classes according to whether they can be written in one or more the following forms [10]:

$$
\begin{gathered}
\rho=\sum_{i}\left|\psi_{i}^{1}\right\rangle\left\langle\psi_{i}^{1}|\otimes| \psi_{i}^{2}\right\rangle\left\langle\psi_{i}^{2}|\otimes| \psi_{i}^{3}\right\rangle\left\langle\psi_{i}^{3}\right|, \\
\rho=\sum_{i}\left|\psi_{i}^{1}\right\rangle\left\langle\psi_{i}^{1}|\otimes| \psi_{i}^{23}\right\rangle\left\langle\psi_{i}^{23}\right| \\
\rho=\sum_{i}\left|\psi_{i}^{2}\right\rangle\left\langle\psi_{i}^{2}|\otimes| \psi_{i}^{13}\right\rangle\left\langle\psi_{i}^{13}\right| \\
\rho=\sum_{i}\left|\psi_{i}^{3}\right\rangle\left\langle\psi_{i}^{3}|\otimes| \psi_{i}^{12}\right\rangle\left\langle\psi_{i}^{12}\right|
\end{gathered}
$$

where $\left|\psi^{1}\right\rangle,\left|\psi^{2}\right\rangle$ and $\left|\psi^{3}\right\rangle$ are states of system 1,2 and 3, respectively, and $\left|\psi^{12}\right\rangle,\left|\psi^{23}\right\rangle$ and $\left|\psi^{13}\right\rangle$ are states of two systems. But no matter how many classes it can be classified into, one can describe it with three cases for convenience: 1) fully seperable states, corresponding to $(3) ; 2)$ incompletely seperable states, corresponding to (4), (5) and (6); 3) fully inseperable states, corresponding to none of above forms.

Considering an $N$-particle pure state no matter which is separable or not , one can always expand it in a series of common basis. If the dimension of the $i$ th subsystem is $D_{i}$, then the dimension of the common basis must be in $\prod_{i} D_{i}$ dimension. Hence, an $\mathrm{N}$-particle state with the fixed dimension of every subsystem can always be converted into a single state with much higher dimension in mathematics. Thus, we can also express an N-particle pure state $\left|\Psi^{A B C \cdots N}\right\rangle$ in $s$ dimension as $\left|\Psi^{A B C \cdots N}\right\rangle=\sum_{i} \sqrt{\lambda_{i}}\left|\Psi_{i}^{1}\right\rangle \otimes\left|\Psi_{i}^{2}\right\rangle$ in terms of the generalized Schmidt decomposition [13], where $\left|\Psi_{i}^{1}\right\rangle$ and $\left|\Psi_{i}^{2}\right\rangle$ are defined in $n_{1}$ and $n_{2}$ dimension, respectively, with $n_{1} \times n_{2}=s$. I.e. an N-particle pure state $\left|\Psi^{A B C \cdots N}\right\rangle$ can always be written as a bipartite state in form, which corresponds to the bipartite grouping of the $N$-particle system. $\left|\Psi_{i}^{1}\right\rangle$ and $\left|\Psi_{i}^{2}\right\rangle$ correspond to each group, respectively. Analogously, an N-particle mixed state $\rho^{A B C \cdots N}=\sum_{i} p_{i}\left|\psi_{i}^{A \cdots N}\right\rangle\left\langle\psi_{i}^{A \cdots N}\right|$ can be operated in the same way because of every pure state $\psi_{i}^{A \cdots N}$. Therefore, considering the bipartite grouping, multiparticle(N-particle) states can also be classified into three classes analogous to three-particle classfication. i.e.

$$
\rho^{A B C \cdots N}=\sum_{i} p_{i}\left|\psi_{i}^{A}\right\rangle\left\langle\psi_{i}^{A}|\otimes \cdots \otimes| \psi_{i}^{N}\right\rangle\left\langle\psi_{i}^{N}\right|
$$




$$
\rho^{A B C \cdots N}=\sum_{i} p_{i}\left|\psi_{i}^{\sum_{j}}\right\rangle\left\langle\psi_{i}^{\sum_{j}}|\otimes| \psi_{i}^{\sum-\sum_{j}}\right\rangle\left\langle\psi_{i}^{\sum_{i}-\sum_{j}}\right|
$$

and

$$
\rho^{A B C \cdots N}=\sum_{i} p_{i}\left|\psi_{i}^{A \cdots N}\right\rangle\left\langle\psi_{i}^{A \cdots N}\right|
$$

where $\sum_{j}=q$ denotes any $q$ subsystems among $A \cdots N, \psi_{i}^{\sum_{j}}$ stands for a common state of $\sum_{j}$ subsystems, $\left|\psi_{i}^{\sum-\sum_{j}}\right\rangle$ stands for the common state of the rest subsystems except the $\sum_{j}$ ones and $\left|\psi_{i}^{A \cdots N}\right\rangle$ denotes a common fully inseparable state of all the $N$ subsystems. If we divide the $\mathrm{N}$ subsystems $\rho^{A B C \cdots N}$ into two big subsystems $\rho^{\sum_{j}}$ and $\rho^{\sum-\sum_{j}}$, one includes one subsystem, i.e. $\sum_{j}=1$ denotes any one of the $\mathrm{N}$ subsystems, and the other includes $\mathrm{N}-1$ subsystems. It is obvious that if

$$
\rho^{A B C \cdots N}=\sum_{i} p_{i} \rho_{i}^{\sum_{j}} \otimes \rho_{i}^{\sum-\sum_{j}}
$$

holds for every $\sum_{j}=1$ (There exist $C_{N}^{1}=N$ ways to realize such a bipartite grouping.), then the whole $\mathrm{N}$-particle system is semiseparable, which was defined in [12]. Note, however, that if there does exist none of all the $\sum_{j}=1$ such that (10) holds, we cannot draw the conclusion that the N-particle system is fully inseparable such as a four-particle pure state $\psi=\psi^{+} \otimes \psi^{+}$, where $\psi^{+}$ is one of the four Bell states. $\rho^{A B C \cdots N}$ may be incompletely separable. Based on the above study, in order to draw a conclusion to estimate whether the $\mathrm{N}$-particle system is fully inseparable, we must enhance the above condition. I.e. $\sum_{j}$ cannot denote only one of the $\mathrm{N}$ subsystems, but every case of $\sum_{j}$ $=1,2, \cdots,\left[\frac{N}{2}\right]$ with $\left[\frac{N}{2}\right]=\left\{\begin{array}{c}N / 2, \quad N \text { is even } \\ (N-1) / 2, N \text { is odd }\end{array}\right.$. However, in order to estimate the inseparability and the semiseparability in the same criterion, we have to express the condition of semiseparability in terms of the condition of full inseparability, which is equivalent to the original one, whilst we express the above conditions in a more rigorous way.

Definition1.- The N-particle system $\rho^{A B C \cdots N}$, which can be divided into two big subsystems $\rho^{\sum_{j}}$ and $\rho^{\sum-\sum_{j}}$ in $\sum_{i=1}^{\left[\frac{N}{2}\right]} C_{N}^{i}$ ways with $C_{N}^{i}=\frac{N !}{(N-i) ! i !}$ and $\sum_{j} \in$ $\left[1,\left[\frac{N}{2}\right]\right]$, is called semiseparable, iff $(10)$ holds for all $\sum_{j} \in\left[1,\left[\frac{N}{2}\right]\right]$, and called fully inseparable, iff $\nexists \sum_{j} \in\left[1,\left[\frac{N}{2}\right]\right]$ such that (10) holds. 


\section{Multiparticle Free Entanglement Measurement}

According to the above redefinition and analysis, we have converted the study of multiparticle inseparabilitiy into study of a series of bipartite inseparability in mathematics. In other words, multiparticle entanglement measure can be obtained from a series of bipartite entanglement measures corresponding to every bipartite grouping. However, note that it does not mean that multiparticle entanglement is equivalent(converted to each other) to bipartite entanglement.

Because the way to divide the whole system to two big subsystems (bipartite grouping) is stochastic and equavilent, the entanglement measure of the whole system is given by

$$
\bar{E}=\sum_{j=1}^{\left[\frac{N}{2}\right]}\left(E_{j} / \sum_{i=1}^{\left[\frac{N}{2}\right]} C_{N}^{i}\right),
$$

where, $\bar{E}$ denotes the multiparticle entanglement measure of the given system and $E_{j}$ denotes the bipartite entanglement measure corresponding to the $j$ th bipartite grouping.

For multiparticle states, one can find that all the bipartite states obtained by our bipartite grouping are pure states or mixed states corresponding to the original pure or mixed multiparticle ones. We have to find an effective bipartite entanglement measure. For bipartite pure states, partial entropy measure or $C(\rho)=\sqrt{2\left(|\langle\psi \mid \psi\rangle|^{2}-\operatorname{Tr} \rho_{r}^{2}\right)}$, the concurrence, which is defined in [14], work well in arbitrary dimension. So $E_{j}=C\left(\rho_{j}\right)$ or $E_{j}=S_{j}\left(\Psi^{12}\right)=-\operatorname{tr}\left\{\rho_{1} \log \rho_{1}\right\}$, where $\rho_{1}=\operatorname{tr}_{2}\left\{\left|\Psi^{12}\right\rangle\left\langle\Psi^{12}\right|\right\}$ is the reduced density matrix and the subscripts 1 and 2 denote the two big subsystems 1 and 2 after bipartite grouping. For bipartite mixed states in higher dimension, it is difficult to find a satisfactory operational entanglement measure. Although that "Concurrence of mixed bipartite quantum states in arbitrary dimensions" [11] proposed recently sheds new light on our problem to some extent, one can also find that this measure is complicated and inoperational. Here, for integrality, we can temporarily employ the concurrence of bipartite mixed state in arbitrary dimension as bipartite entanglement measure. I.e. $E_{j}=c\left(\rho_{j}\right)$ with $\rho_{j}$ standing for the bipartite density matrix by the bipartite grouping in the $j$ th way. In some cases, in order to give an explicit expression of entanglement measure of a state, we can also employ the negativity $N(\rho)=\frac{\left\|\rho^{T} A\right\|-1}{2}$ defined in [15], which corresponds to the absolute value of the sum of negative eigenvalues of $\rho^{T_{A}}[17]$. Of course, a better bipartite entanglement measure will be expectable and better compensative for our measure. But no matter which measure one chooses, in the same case, one must employ the same measure as must work without any mistake in the given case. 
In our multiparticle entanglement measure, we have to divide the whole Nparticle system into two big subsystems by virtue of the above method. Equivalently, we can construct a series of permutation operations to realize the bipartite grouping. Consider $\rho^{A B C \cdots N}=\sum_{i} p_{i}\left|\psi_{i}^{A B \cdots N}\right\rangle\left\langle\psi_{i}^{A B \cdots N}\right|$ in $s$ dimension, with subsystem $A$ and subsystem $B$ in $n_{1}$ and $n_{2}$ dimension respectively, we can get that

$$
\begin{gathered}
\rho^{B(A C \cdots N)}=\sum_{i} p_{i}\left|\psi_{i}^{B(A C \cdots N)}\right\rangle\left\langle\psi_{i}^{B(A C \cdots N)}\right| \\
=\sum_{i} p_{i}\left(P\left(n_{1}, n_{2}\right)^{T} \otimes 1^{C \cdots N}\right)\left|\psi_{i}^{A B \cdots N}\right\rangle \times\left\langle\psi_{i}^{A B \cdots N}\right|\left(P\left(n_{1}, n_{2}\right) \otimes 1^{C \cdots N}\right) \\
=\left(P\left(n_{1}, n_{2}\right)^{T} \otimes 1^{C \cdots N}\right) \rho^{A(B C \cdots N)}\left(P\left(n_{1}, n_{2}\right) \otimes 1^{C \cdots N}\right),
\end{gathered}
$$

where the bracket in the superscripts denotes a whole subsystem, $P\left(n_{1}, n_{2}\right)$ is permutation matrix defined as

$$
P\left(n_{1}, n_{2}\right)=\sum_{i=1}^{n_{1}} \sum_{j=1}^{n_{2}} E_{i j} \otimes E_{i j}^{T}=\left(\begin{array}{cccc}
E_{11}^{T} & E_{12}^{T} & \cdots & E_{1 n_{2}}^{T} \\
E_{21}^{T} & E_{22}^{T} & \cdots & E_{2 n_{2}}^{T} \\
\vdots & \vdots & \ddots & \vdots \\
E_{n_{1} 1}^{T} & E_{n_{1} 2}^{T} & \cdots & E_{n_{1} n_{2}}^{T}
\end{array}\right)
$$

$E_{i j}$ is a matrix in $n_{1} \times n_{2}$ dimension with subscript $i j$ denoting the matrix element $e_{i j}=1$ and the rests are zero in the matrix $E_{i j}$. By such a transformation, an $n_{1} \times\left(s / n_{1}\right)$ bipartite $\rho^{A(B C \cdots N)}$ is transformed to an $n_{2} \times\left(s / n_{2}\right)$ bipartite $\rho^{B(A C \cdots N)}$. In terms of dividing the whole system in $\sum_{i=1}^{\left[\frac{N}{2}\right]} C_{N}^{i}$ ways, we have to construct corresponding permutation matrix with the same quality. Generally, we first construct a permutation which moves the $j$ th particle to the position of the $i$ th one and moves the $i$ th particle to the position of the $(i+1)$ th one as

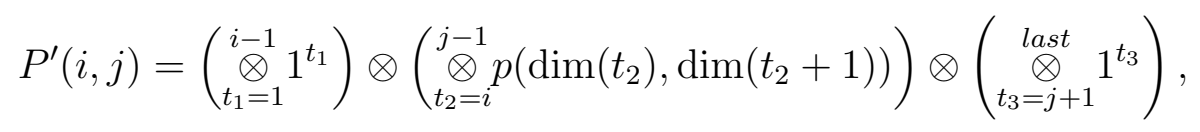

here $1^{\alpha}$ stands for unit matrix with the same dimension to the $\alpha$ th particle, $\operatorname{dim}(i)$ denotes the dimension of the $i$ th particle and $\underset{t_{1}=1}{\otimes} 1^{t_{1}}=1$. What's more, we require that $\underset{t_{2}=i}{\otimes+1} p\left(\operatorname{dim}\left(t_{2}\right), \operatorname{dim}\left(t_{2}+1\right)\right)=p(\operatorname{dim}(i), \operatorname{dim}(i+1)) \otimes p(\operatorname{dim}(i+$

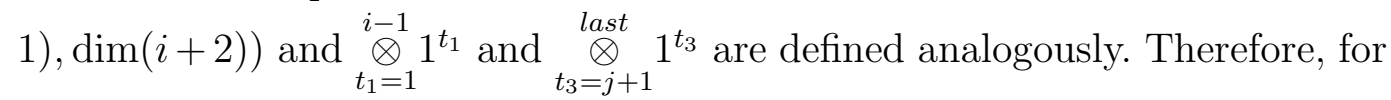
the $k$ th grouping, one of the two big subsystems includes $M$ particles each of which lies on the $X_{i}$ th position, we can construct a unitary transformation by the permutation to realize the grouping as

$$
U_{k}=\prod_{i=1}^{N} P^{\prime}\left(i, X_{i}\right)
$$


analogously, $\prod_{i=j}^{j+1} P^{\prime}\left(i, X_{i}\right)=P^{\prime}\left(j, X_{j}\right) \times P^{\prime}\left(j+1, X_{j+1}\right)$. Note that the order of every particle in each big subsystem does not influence the separability relation between the two big subsystems. The different orders are just like local unitary transformations. If we operate every $U_{k}$ on the density matrix $\rho^{A B C \cdots N}$, we get a density matrix $\rho_{k}=U_{k}^{T} \rho^{A B C \cdots N} U_{k}$ according to the $k$ th grouping. Thus we can get a set $\rho=\left\{\rho_{k} \mid k=0,1, \cdots \sum_{i=1}^{\left[\frac{N}{2}\right]} C_{N}^{i}\right\}$, every element of which corresponds to every $E_{j}$ in $(10)$.

\section{Examples}

As examples, for pure states, consider a general three-particle pure state

$$
\begin{gathered}
\left|\Psi^{A B C}\right\rangle=\left(C_{1}|0\rangle_{A}+C_{2}|1\rangle_{A}\right)\left|\phi_{B C}^{+}\right\rangle+\left(C_{3}|0\rangle_{A}+C_{4}|1\rangle_{A}\right)\left|\phi_{B C}^{-}\right\rangle \\
+\left(C_{5}|0\rangle_{A}+C_{6}|1\rangle_{A}\right)\left|\psi_{B C}^{+}\right\rangle+\left(C_{7}|0\rangle_{A}+C_{8}|1\rangle_{A}\right)\left|\psi_{B C}^{-}\right\rangle,
\end{gathered}
$$

with $\sum_{i=1}^{8}\left|C_{i}\right|^{2}=1,\left|\phi_{B C}^{ \pm}\right\rangle=\frac{1}{\sqrt{2}}(|00\rangle \pm|11\rangle)$ and $\left|\psi_{B C}^{ \pm}\right\rangle=\frac{1}{\sqrt{2}}(|01\rangle \pm|10\rangle)$. By our entanglement measure, $\sum_{i=1}^{\left[\frac{3}{2}\right]} C_{3}^{i}=C_{3}^{1}=3$, and

$$
C\left(\rho_{i}\right)=\sqrt{2\left(|\langle\psi \mid \psi\rangle|^{2}-\operatorname{Tr}\left(\rho_{i}\right)_{r}^{2}\right)}=\sqrt{2\left(1-\operatorname{Tr}\left(\rho_{i}\right)_{r}^{2}\right)}
$$

with $i$ denoting $A-B C, B-A C$ and $C-A B$, three different groupings respectively. Hence, we have

$$
\bar{E}=\frac{1}{3} \sum_{i} C\left(\rho^{i}\right)=\frac{1}{3}\left[C\left(\rho^{A-B C}\right)+C\left(\rho^{B-A C}\right)+C\left(\rho^{C-A B}\right)\right],
$$

with

$$
C\left(\rho^{i}\right)=\sqrt{2\left(1-\left(M_{i}^{2}+N_{i}^{2}+2 P_{i} Q_{i}\right)\right)}
$$

where

$$
\begin{gathered}
M_{A-B C}=\left|C_{1}\right|^{2}+\left|C_{3}\right|^{2}+\left|C_{5}\right|^{2}+\left|C_{7}\right|^{2}, \\
N_{A-B C}=\left|C_{2}\right|^{2}+\left|C_{4}\right|^{2}+\left|C_{6}\right|^{2}+\left|C_{8}\right|^{2}, \\
P_{A-B C}=C_{1} C_{2}^{*}+C_{3} C_{4}^{*}+C_{5} C_{6}^{*}+C_{7} C_{8}^{*}, \\
M_{B-A C}=\frac{1}{2}\left(\left|C_{1}+C_{3}\right|^{2}+\left|C_{2}+C_{4}\right|^{2}+\left|C_{5}+C_{7}\right|^{2}+\left|C_{6}+C_{8}\right|^{2}\right), \\
N_{B-A C}=\frac{1}{2}\left(\left|C_{1}-C_{3}\right|^{2}+\left|C_{2}-C_{4}\right|^{2}+\left|C_{5}-C_{7}\right|^{2}+\left|C_{6}-C_{8}\right|^{2}\right),
\end{gathered}
$$




$$
\begin{aligned}
& P_{B-A C}= \frac{1}{2}\left(\left(C_{1}+C_{3}\right)\left(C_{5}-C_{7}\right)^{*}+\left(C_{5}+C_{7}\right)\left(C_{1}-C_{3}\right)^{*}\right. \\
&+\left(C_{2}+C_{4}\right)\left(C_{6}-C_{8}\right)^{*}+\left(C_{6}+C_{8}\right)\left(C_{2}-C_{4}\right)^{*} \\
& M_{C-A B}= \frac{1}{2}\left(\left|C_{1}+C_{3}\right|^{2}+\left|C_{2}+C_{4}\right|^{2}+\left|C_{5}-C_{7}\right|^{2}+\left|C_{6}-C_{8}\right|^{2}\right), \\
& N_{C-A B}=\frac{1}{2}\left(\left|C_{1}-C_{3}\right|^{2}+\left|C_{2}-C_{4}\right|^{2}+\left|C_{5}+C_{7}\right|^{2}+\left|C_{6}+C_{8}\right|^{2}\right), \\
& P_{C-A B}=\frac{1}{2}\left(\left(C_{1}+C_{3}\right)\left(C_{5}+C_{7}\right)^{*}+\left(C_{5}-C_{7}\right)\left(C_{1}-C_{3}\right)^{*}\right. \\
&+\left(C_{2}+C_{4}\right)\left(C_{6}+C_{8}\right)^{*}+\left(C_{6}-C_{8}\right)\left(C_{2}-C_{4}\right)^{*} \\
& Q_{i}=P_{i}^{*} .
\end{aligned}
$$

Therefore, we have given out the entanglement measure of all the three-particle pure states. one can evaluate $\bar{E}$ according to the given quantum state. For example, consider $C_{1}=C_{4}=\frac{1}{\sqrt{2}}$ and the rest are zero, then $\bar{E}=1$ by our measure, which suggests $\left|\Psi^{A B C}\right\rangle$ is a GHZ state. Substitute $C_{1}$ and $C_{4}$ to (15), then $\left|\Psi^{A B C}\right\rangle=\frac{1}{2}(|0\rangle+|1\rangle)^{A}|00\rangle^{B C}+\frac{1}{2}(|0\rangle-|1\rangle)^{A}|11\rangle^{B C}$ which is only a local unitary transformation different from the GHZ state, $\left|\Psi^{\prime} A B C\right\rangle=$ $\frac{1}{\sqrt{2}}|000\rangle^{A B C}+\frac{1}{\sqrt{2}}|111\rangle^{A B C}$. This result is consistent to our measure.

For mixed states, consider such a state as

$$
\rho=x\left|\psi_{0}^{+}\right\rangle\left\langle\psi_{0}^{+}\right|+\frac{1-x}{2^{N}} 1
$$

which is described in $[10,16]$. We employ the negativity $N(\rho)$ as entanglement measure. By our method, one can find that the $N$-particle state can be divided in $\sum_{i=1}^{\left[\frac{N}{2}\right]} C_{N}^{i}$ ways, and $N\left(\rho_{j}\right)=\left|\frac{1-\left(1+2^{N-1}\right) x}{2^{N}}\right|$ for $j=1,2, \cdots \sum_{i=1}^{\left[\frac{N}{2}\right]} C_{N}^{i}$ and $x>$ $\frac{1}{\left(1+2^{N-1}\right)}$ with subscript $j$ denoting the bipartite grouping in the $j$ th way. Hence one can get

$$
\bar{E}(\rho)=\left\{\begin{array}{cc}
\left|\frac{1-\left(1+2^{N-1}\right) x}{2^{N}}\right|, & x>\frac{1}{\left(1+2^{N-1}\right)} \\
0 & \text { otherwise }
\end{array} .\right.
$$

This result is not only consistent to the previous one [10], but also gives an explict entanglement measure for $x>\frac{1}{\left(1+2^{N-1}\right)}$. 


\section{Conclusion}

In conclusion, we have shown a redefinition to estimate whether a multiparticle system is semiseparable or fully inseparable. In terms of the redefinition, we convert multiparticle entanglement measure into a series of bipartite entanglement measures in mathematics and then give out the multiparticle entanglement measure with a simple form $\bar{E}=\sum_{j=1}^{\sum_{i=1}^{\left[\frac{N}{2}\right]} C_{N}^{i}}\left(E_{j} / \sum_{i=1}^{\left[\frac{N}{2}\right]} C_{N}^{i}\right)$. At last, we give two examples demonstrating that our measure is reasonable and feasible. Due to the convenient and operational bipartite entanglement measure for pure states, our measure for multiparticle entanglement works better, unlike the rather cumbersome measure for mixed states. But in some special cases, it can also work better to employ a special measure for bipartite states, so long as the bipartite measure can work without any mistake. What's more, we carry out all our studies about the inseparability and semiseparability of a state in mathematics, and we think the inseparbility measure as entanglement measure analogous to bipartite entanglement, but it is doesn't mean that the multiparticle entanglement and bipartite entanglement are equivalent(converted to each other) in physics.

\section{Acknowledgement}

We thank X. X. Yi for extensive and valuable advice. This work was supported by Ministry of Science and Technology, China, under grant No.2100CCA00700.

\section{References}

[1] C.H.Bennett,et al.,Phys.Rev.Lett.70,1895 (1993).

[2] M. Zukowski, A. Zeilinger, M. A. Horne, and A. K. Ekert, Phys. Rev. Lett. 71, 4287 (1993).

[3] C.Bennett and G.Brassard, in Proc. of IEEE, pp.175-179 (1984).

[4] V.Coffman, J.Kundu, and W. K. Wootters, Phys. Rev. A 61, 052306 (2000).

[5] W. K. Wootters, Phys.Rev. Lett. 80, 2245 (1998).

[6] G.Vidal, J.Mod.Opt. 47, 355 (2000).

[7] C.H.Bennett, D.P.DiVicenzo,J.Smolin, and W. K. Wootters, Phys.Rev.A 54,3824(1996).

[8] V.I. Yukalov, Phys. Rev. Lett. 90, 167905 (2003).

[9] Alexander Wong and Nelson Christensen, Phys.Rev.A 63, 044301 (2001). 
[10] W.Dür,J.I.Cirac and R.Tarrach, Phys.Rev. Lett. 83, 3562 (1999).

[11] Florian Mintert, Marek Kuś, and Andreas Buchleitner, Phys.Rev. Lett. 92, 167902 (2004).

[12] PAWEL HORODECKI and RYSZARD HORODECKI, Quantum Information and Computation 1, No.1 45 (2001).

[13] M.Hossein Partovi, Phy.Rev. Lett. 92, 077904 (2004).

[14] Pranaw Rungta, V. Bužek, Carlton M. Caves, M. Hillery, and G. J. Milburn, Phy.Rev. A 64, 042315 (2001).

[15] G.Vidal and R.F.Werner, Phys.Rev.A 65,032314(2002).

[16] G.Vidal and R.Tarrach, Phys.Rev.A 59,141(1999).

[17] K.Zyczkowski, P. Horodecki, A.Sanpera, and M.Lewenstein, Phys. Rev. A 58, 883(1998); B.K.Zyczkowski, Phys. Rev. A 60, 3496 (1999). 